$\Rightarrow$ MYOCARDITIS

Midkine promotes NETosis in myocarditis

The cytokine midkine promotes trafficking of polymorphonuclear neutrophils into the myocardium and the formation of neutrophil extracellular traps (NETs) in myocarditis, according to new research published in the Journal of Experimental Medicine. "Whereas the role of adaptive immunity in myocarditis has been studied quite extensively ... the contribution of the innate immune system and in particular neutrophils has been only marginally addressed," comments Ludwig Weckbach, the first author on the paper.

The researchers identified, for the first time, NETs in endomyocardial biopsies from patients with myocarditis and in cardiac tissue from mice with experimental autoimmune myocarditis. NETs are formed when neutrophils are stimulated (for example, by viruses, bacteria or cytokines) to eject their DNA decorated with antimicrobial proteins, generating inflammation and tissue injury. Inhibition of NETosis in mice with myocarditis substantially reduced inflammation in the acute phase of the disease.

Midkine is a $13-k D$ cytokine that is critical for neutrophil adhesion and subsequent extravasation during acute inflammation. In mice with myocarditis, inhibiting midkine with a blocking antibody had the dual effects of attenuating both neutrophil recruitment and NET formation. The mechanism by which midkine drives neutrophil recruitment and NETosis is likely to be via the low-density lipoprotein receptor-related protein 1 , which is expressed on neutrophils.

"To date, there is no established therapy for patients with chronic inflammatory cardiomyopathy other than nonspecific immunosuppression with limited benefits and severe side effects," state the researchers. "Therefore, blocking midkine or NETs could represent novel and promising therapeutic options for patients with myocarditis." Weckbach and colleagues also plan to investigate whether their findings are applicable to autoimmune myocarditis triggered by the use of checkpoint inhibitors in the treatment of cancer.

Gregory B. Lim

ORIGINAL ARTICLE Weckbach, L. T. et al. Midkine drives cardiac inflammation by promoting neutrophil trafficking and NETosis in myocarditis.J.Exp. Med. https://doi.org/10.1084/ jem.20181102(2019)

\title{
Poor sleep linked to atherosclerosis
}

According to a new study, shorter sleeping times and fragmented sleep are associated with an increased risk of subclinical atherosclerosis. Epidemiological studies had already linked short sleep duration to a higher risk of cardiovascular disease (CVD), but most of these studies relied on self-reported sleep evaluation.

"The aim of this sleep study was to evaluate the association between objective actigraphy-measured sleep parameters and subclinical atherosclerosis using state-of-the-art imaging techniques," explains lead investigator José Ordovás. A total of 3,974 participants from the PESA CNIC-Santander study - a Spanish observational, prospective cohort on the progression of subclinical CVD were included in the study. Vascular ultrasonography and cardiac CT were used to assess noncoronary atherosclerosis and coronary calcification, and 7-day actigraphic recording was performed to measure sleep parameters. Four groups were defined according to sleep duration: very short sleep $(<6 \mathrm{~h})$, short sleep $(6-7 \mathrm{~h})$, reference sleep $(7-8 \mathrm{~h})$ and long sleep $(>8 \mathrm{~h})$.
After adjustment for traditional risk factors, very short sleep duration was associated with increased atherosclerotic burden compared with the reference group (OR 1.27, 95\% CI 1.06-1.52, $P=0.008$ ). Sleep quality was assessed by the total sleep fragmentation index; participants with the most fragmented sleep had the highest risk of multiple affected noncoronary territories compared with the reference group with the least fragmented sleep (OR 1.34, 95\% CI 1.09-1.64, $P=0.006)$. No differences were seen in coronary artery calcification scores between the different sleep groups.

"These significant and robust findings open new areas of research, including investigating whether an increase in the amount and quality of sleep decreases the development of atherosclerosis in people with sleep deficiencies," concludes Ordovás.

Alexandra Le Bras

ORIGINAL ARTICLE Domínguez, F. et al. Association of sleep duration and quality with subclinical atherosclerosis. J. Am. Coll. Cardiol. 73, 134-144 (2019)

FURTHER READING Tobaldini, E. et al. Short sleep duration and cardiometabolic risk: from pathophysiology to clinical evidence. Nat. Rev. Cardiol. https://doi.org/10.1038/ s41569-018-0109-6 (2018)

\section{Clonal haematopoiesis predicts poor prognosis in heart failure}

Clonal haematopoiesis of indeterminate potential (CHIP) is the clonal expansion of haematopoietic cells caused by somatic mutations. This phenomenon has been shown to increase with age and to be associated with inflammation and atherosclerosis. A new study published in JAMA Cardiology now links CHIP with poor prognosis in chronic heart failure (HF) of ischaemic origin.

Bone-marrow-derived mononuclear cells were obtained from 200 patients with chronic $\mathrm{HF}$ and analysed by deep targeted amplicon sequencing to detect the presence of CHIP. A total of 47 mutations with a variant allele fraction $\geq 0.02$ were found in 38 of the patients (18.5\%). The somatic mutations most commonly occurred in DNMT3A (14 patients), TET2 (nine patients), KDM6A (four patients) and BCOR (three patients).

DNMT3A and TET2 encode proteins involved in DNA methylation and demethylation, which exert epigenetic control on gene expression. Functionally, these two proteins are important regulators of inflammation by augmenting monocyte adhesion and macrophage activity. Patients with either DNMT3A or TET2 mutations had significantly worse long-term clinical outcomes than patients who were not CHIP carriers. The presence of DNMT3A or TET2 mutations was independently associated with an increased risk of death or hospitalization for HF (HR 2.1, 95\% Cl 1.1-4.0, $P=0.02$ ). Of note, a significant dose-response association was reported between variant allele fraction and clinical outcome.

The investigators speculate that identifying patients with $\mathrm{HF}$ who are $\mathrm{CHIP}$ carriers could inform a precision-medicine approach. For example, antioxidants might be used to activate remaining wild-type TET2 in patients with TET2 mutations.

Gregory B. Lim

ORIGINAL ARTICLE Dorsheimer, L. et al. Association of mutations contributing to clonal hematopoiesis with prognosis in chronic ischemic heart failure. JAMA Cardiol. https://doi.org/10.1001/jamacardio.2018.3965 (2018) 